\title{
Sexual Recombinants Make a Significant Contribution to Epidemics Caused by the Wheat Pathogen Phaeosphaeria nodorum
}

\author{
Rubik J. Sommerhalder, Bruce A. McDonald, Fabio Mascher, and Jiasui Zhan
}

First and second authors: Plant Pathology, Institute of Integrative Biology, ETH Zurich, LFW, Universitaetstrasse 2, CH-8092 Zürich, Switzerland; third author: Swiss Federal Research Station for Agronomy, Changins, CH-1260 Nyon, Switzerland; and fourth author: Industrial Crop Research Institute, Yunnan Academy of Agricultural Sciences, Longtou Street, Kunming, 650205, People's Republic of China.

Accepted for publication 28 April 2010.

\begin{abstract}
Sommerhalder, R. J., McDonald, B. A., Mascher, F., and Zhan, J. 2010. Sexual recombinants make a significant contribution to epidemics caused by the wheat pathogen Phaeosphaeria nodorum. Phytopathology 100:855-862.

We conducted a 2-year mark-release-recapture field experiment to quantify the relative contributions of immigration and sexual and asexual reproduction to epidemics of Stagonospora nodorum blotch caused by Phaeosphaeria nodorum. The epidemic was initiated using nine geneti-

The proportion of isolates having multilocus haplotypes (MLHTs) identical to the inoculated isolates decreased steadily from $86 \%$ in the first collection to $25 \%$ in the fourth collection. The novel isolates that had different MLHTs compared with the marked inoculants originated through immigration and sexual recombination. By the end of the experiment, nearly three-quarters of the novel isolates originated from sexual recombination. Our results indicate that recombinant offspring and airborne immigrant ascospores can make significant contributions to epidemics of Stagonospora nodorum blotch during a growing season.
\end{abstract} cally distinct $P$. nodorum isolates. Infected plants were sampled four times across two growing seasons. In total, 1,286 isolates were recovered and assayed with 10 microsatellite markers and 1 minisatellite marker.
Additional keywords: Bayesian theory, maximum likelihood estimation, population genetics, primary inoculum.
Reproductive mode has important epidemiological consequences for many plant pathogens (33). Some plant pathogenic ascomycetes, such as Phaeosphaeria nodorum, produce splashdispersed asexual conidia that move only short distances (1 to $2 \mathrm{~m}$ ) over the course of a growing season, leading to spatially limited foci of infection for individual clones. Through sexual reproduction, $P$. nodorum also produces airborne ascospores $(3,4,13)$ that have the ability to travel long distances $(12,18,50)$, potentially leading to rapid spread of selected traits such as novel virulence across large regions (55). Sexual reproduction also increases genetic variation in populations by generating new alleles through intragenic recombination $(10,42,56,57)$ as well as novel allele combinations (multilocus genotypes) by shuffling existing alleles. The combination of high gene flow potential (e.g., through the production of air-dispersed ascospores) and increased genetic variation (e.g., through undergoing regular cycles of sexual recombination) may allow pathogens with sexual reproduction to evolve and adapt more rapidly to changing environments, reducing the useful life span of fungicides and resistant cultivars (29) and thereby making disease control more difficult.

Combining neutral molecular markers with analytical tools of population genetics has provided a powerful approach to infer the reproductive biology and epidemiological properties of many fungal pathogens for which sexual phases are known or unknown $(11,25,27,34,37,60)$. However, very few studies have combined these tools to directly quantify the relative contributions of sexual reproduction, asexual reproduction, and immigration to the development of epidemics under field conditions $(1,5,61,62,64)$.

Corresponding author: J. Zhan; E-mail address: Jiasui.zhan@yahoo.com

doi:10.1094/PHYTO-100-9-0855

(C) 2010 The American Phytopathological Society
The heterothallic loculoascomycete $P$. nodorum (E. Müll.) Hedjar. (syn. Septoria nodorum (Berk.) Berk.), the teleomorph form of Stagonospora nodorum (Berk.) E. Castell. \& Germano (syn. Leptosphaeria nodorum E. Müll.), causes Stagonospora nodorum blotch on wheat (Triticum aestivum L.). It is a significant wheat pathogen globally (24). The teleomorph has been observed in many locations around the world $(3,4,13$, $14,19,23,32,38,43)$ and the primary inoculum of the disease has been thought to be composed mainly of sexually produced, winddispersed ascospores $(21,22)$, asexually infected seed (44), and crop residue (20). Although many studies $(2,7,21,46-48,52)$ indicated that sexual reproduction plays an important role in the population genetic structure of the pathogen, successful recovery of a large proportion of noninoculated genotypes (43\%) from a mark-release-recapture field experiment conducted in North America (5) provided only indirect evidence that ascospores also can make a significant contribution to epidemic development during a growing season. Though it is known that the fungus can undergo both asexual and sexual reproduction, it is not clear how often and when the latter occurs. The most common assumption is that the sexual cycle occurs on infected straw residue left in the field between growing seasons, with the release of ascospores during autumn and spring coinciding with the emergence of seedlings in winter wheat $(4,5,35,38,43)$. We hypothesized that ascospores could play a significant role in the development of Stagonospora nodorum leaf blotch both through initiating the epidemic (i.e., as primary inoculum) and by spreading the disease within and among fields during the growing season (i.e., as secondary inoculum). The objective in this experiment was to elucidate the relative contributions of different sources of inoculum to the population genetic structure and development of $P$. nodorum on wheat. To achieve this objective, we conducted a 2-year field experiment using a mark-release-recapture experimental design. The isolates recovered from infected leaves at 
different sampling times were assigned to different categories of source inoculum, namely asexual progeny of the released inoculants, immigrants from surrounding regions, or recombinants arising from within the experimental plots, using a combination of molecular genotyping, maximum likelihood estimates, and posterior probabilities.

\section{MATERIALS AND METHODS}

Experimental design. A field experiment was conducted in two fields at the Agroscope research center in Changins, Switzerland during the 2003-04 and 2004-05 winter wheat seasons. The two fields were $\approx 25 \mathrm{~m}$ apart and both of them were covered with a perennial meadow for at least 3 years prior to the experiment. Four commercial Swiss wheat cultivars (Levis, Runal, Tamaro, and Tirone) were used in this experiment. The four cultivars and a 1:1 mixture of Runal and Tamaro (five host treatments in total) were planted in a randomized complete block design with three replications in the main part of the experiment. These plots (hereafter called the inoculated plots) were inoculated with nine known $P$. nodorum strains. Another replication of the five host treatments was planted next to the main experiment; however, these plots (hereafter called the noninoculated control plots) received no artificial inoculation. Because isolates collected from the noninoculated control plots resulted from natural infection, these isolates could be used to estimate allele frequencies in the local $P$. nodorum population. Both the inoculated and noninoculated control plots were $1.5 \mathrm{~m}$ wide and $4.5 \mathrm{~m}$ long. Each wheat plot was surrounded by four equal-sized plots planted at the same time with the highly resistant winter triticale cv. Tridel. The experiment was planted on 5 October 2003 in the first year and on 17 October 2004 in the second year using commercial seed treated with Coral $(2.38 \%$ difenoconazole and $2.38 \%$ fludioxonil, $2 \mathrm{ml} / \mathrm{kg}$ of seed). The experimental field was surrounded by soybean crops and meadows during the 2003-04 season and by sunflower, oilseed rape, and meadows during the 2004-05 season. The distance to the nearest wheat field was $\approx 200 \mathrm{~m}$.

Nine $P$. nodorum isolates originating from naturally infected fields near Bern, Switzerland in 1999 were chosen as inoculants for the 2003-04 experiment. Each of the isolates had distinct multilocus haplotypes (MLHTs) when assayed with 10 polymorphic microsatellite markers and one minisatellite marker (Table 1) (53). PCR amplifications of the mating type idiomorphs $(6,47)$ showed that five of the nine isolates were MAT1-1 and the other four were MAT1-2. The isolates were first grown on yeast maltose agar (yeast at $4 \mathrm{~g} \mathrm{liter}^{-1}$, maltose at $4 \mathrm{~g} \mathrm{liter}^{-1}$, sucrose at $4 \mathrm{~g} \mathrm{liter}^{-1}$, and agar at $10 \mathrm{~g} \mathrm{liter}^{-1}$ ) at $21^{\circ} \mathrm{C}$ for 10 days and then transferred to 1,000-ml flasks containing $300 \mathrm{~g}$ of sterilized wheat kernels (cv. Arina) in a dark incubator at $4^{\circ} \mathrm{C}$. Three months later, the infected wheat kernels were harvested and ground to a powder using a gristmill. The powdered kernels were mixed with distilled water and the spore suspension was filtered through cheesecloth and glass-wool. The spore suspension from each isolate was adjusted to $10^{6}$ spores $/ \mathrm{ml}$ using a hemacytometer and the spores from each isolate were mixed in equal proportions. A surfactant (Tween 20) was added to the spore suspension at the rate of one drop per $50 \mathrm{ml}$. The aqueous spore suspension was applied with a Birchmeyer backpack sprayer (Birchmeyer Spritztechnik AG, Stetten, Switzerland) as uniformly as possible onto wheat seedlings in the inoculated plots at growth stage 31 on 11 May 2004. Plots were checked for disease symptoms once each week following seedling emergence but no disease was observed in any of the plots at the time of inoculation. Each field plot was sprayed with $500 \mathrm{ml}$ of the calibrated spore suspension. To optimize the humidity and increase the efficiency of infection, inoculations were carried out in the late afternoon on a cloudy day and the inoculated seedlings were covered with plastic tarps for $24 \mathrm{~h}$. The source of primary inoculum in the 2004-05 experiment was the infected straw and other plant debris saved from the first year's experiment. After harvesting the grain at the end of July 2004, the straw and other plant debris in each plot were collected and stored separately in burlap potato sacks for 3 months. The sacks were stored in a dry, dark, cool room to allow the development of a saprophytic phase without the risk of excessive molding. At the beginning of tillering (Zadoks stage 13 to 21), the straw was applied onto the corresponding host plots.

In total, four fungal collections were made from both the inoculated and noninoculated control plots during the two growing seasons. The first collection was made on 4 June 2004 from the third or fourth full leaf at 3 weeks after the artificial inoculation. The second collection was made on 2 July 2004 from flag leaves. The third collection (from the second true leaf) was made on 11 April 2005 and the last collection (from the third true leaf) was made on 10 June 2005. For each collection, 30 to 40 leaves were collected from each inoculated plot at intervals of $\approx 20 \mathrm{~cm}$ along transects within the inner rows of the field plots. In most cases, only one isolation was made from each infected leaf. However, because few pycnidia were found in lesions on many of the leaves, the total number of isolations made was much lower than the number of wheat leaves collected. For some collections with very low levels of infection, two isolations were made from the same leaf. In these cases, each isolate was obtained from clearly separated lesions to minimize the possibility of sampling the same infection event. Our earlier work showed that $P$. nodorum isolations made from different lesions within an infected leaf usually contain different genotypes, suggesting that they originate from different infection events $(31,47)$.

DNA extraction and microsatellite data collection. DNA was extracted from each isolate using the DNeasy Plant Mini DNA extraction kit (Qiagen $\mathrm{GmbH}$, Germany) according to the specifications of the manufacturer. The genotype of each isolate was determined using the same minisatellite and microsatellite markers used to tag the released isolates (Table 1). Multiplexed polymerase chain reactions (PCR) were carried out with fluorescently labeled primers using the same conditions described previously (53). Sizes of amplicons were determined using an ABI PRISM 3100 sequencer in which a DNA size ladder was included in each of the samples. Alleles were assigned using the program Genescan (version 3.7; Applied Biosystems) with a binning procedure.

TABLE 1. Microsatellite and mating type alleles for the nine Phaeosphaeria nodorum isolates used in the field experiment

\begin{tabular}{|c|c|c|c|c|c|c|c|c|c|c|c|c|}
\hline Isolate & SN15 & SN3 & SN11 & SN5 & SN1 & SN17 & SN8 & SN22 & SN21 & SN23 & SN16 & Mating type \\
\hline SN99CH2.04a & 162 & 303 & 168 & 426 & 286 & 128 & 403 & 244 & 212 & 312 & 191 & 1 \\
\hline SN99CH2.09a & 164 & 303 & 168 & 447 & 289 & 98 & 364 & 250 & 203 & 363 & 201 & 2 \\
\hline SN99CH2.12a & 164 & 303 & 168 & 429 & 283 & 98 & 403 & 247 & 203 & 312 & 191 & 1 \\
\hline SN99CH3.08a & 168 & 303 & 168 & 426 & 287 & 107 & 364 & 238 & 200 & 312 & 191 & 1 \\
\hline SN99CH3.09a & 164 & 303 & 168 & 429 & 287 & 101 & 364 & 247 & 212 & 312 & 201 & 1 \\
\hline SN99CH3.10a & 164 & 303 & 168 & 429 & 287 & 101 & 364 & 244 & 212 & 312 & 191 & 2 \\
\hline SN99CH3.20a & 164 & 303 & 168 & 429 & 298 & 98 & 364 & 238 & 203 & 312 & 201 & 2 \\
\hline SN99CH3.23a & 164 & 303 & 168 & 429 & 298 & 98 & 364 & 244 & 236 & 312 & 201 & 2 \\
\hline $\mathrm{C} 1$ & 164 & 306 & 168 & 441 & 286 & 98 & 403 & 244 & 206 & 312 & 201 & 1 \\
\hline
\end{tabular}


Data analysis. Allele information was combined across the 11 marker loci to form an MLHT for each isolate. Isolates with the same MLHT as the inoculated genotypes were considered to be the asexual progeny of these inoculated genotypes (hereafter called inoculants). The probability that two isolates will have the same MLHT by chance was calculated as described earlier (30) using the frequencies of the microsatellite and minisatellite alleles observed in this experiment. If the frequency of allele $i$ at locus $j$ be $P_{i j}$, then the probability that two isolates will have the same MLHT haplotype (i.e., that both isolates will contain the $i$ th allele at the $j$ th locus across all loci by chance) will be

$$
\prod_{1}^{j} P_{i j}
$$

The highest and lowest probability of having the same MLHT by chance will be found in the isolates containing the most common and rarest alleles across all loci, respectively. Using this method, we estimated that the probability that two isolates having the same MLHT by chance ranged from $6.3 \times 10^{-5}$ to $3.8 \times 10^{-14}$. Unless specifically mentioned, only novel isolates, defined as isolates having MLHTs different from any of the nine inoculants, were included in the estimates of genetic parameters throughout this manuscript. Because the contribution of mutation to the formation of new genotypes is expected to be trivial within the time scale of this experiment, these novel isolates could originate either via immigration from outside of the experimental plots or by recombination among inoculants or immigrants within the experimental plots.

Populations sampled from the inoculated plots were grouped chronologically and, hereafter, are called 2004A for the first collection, 2004B for the second collection, 2005A for the third collection, and 2005B for the fourth collection. Due to the limited number of isolates recovered from the noninoculated control plots, isolates from all four collections were pooled to form a single population called "Control". Analyses of allele frequencies and gametic disequilibria were performed on clone-corrected data using a single representative of isolates having the same MLHT. Genotypic diversity was quantified using standardized Stoddart and Taylor's measurement of diversity (51) as described previously (16). Comparisons of allele frequencies between noninoculated control and inoculated plots were based on a contingency $\chi^{2}$ test as described by Everitt (15). Gametic disequilibria in 2004B, 2005A, 2005B, and Control were evaluated using the multilocus association test (8) implemented in POPGENE32 (59) and the Phylogenetic Tree Length Permutation Test $(11,28)$ implemented in PAUP 4.0 (54). Tests for gametic disequilibrium were not made for 2004A due to its small sample size (20 novel genotypes).

The prior probabilities of novel isolates originating from sexual reproduction among fungal isolates within the host canopy or via immigration from outside the host canopy were estimated with a likelihood method described previously (63). The allele frequencies estimated from all isolates (novel + inoculated) in an inoculated plot were used to calculate the probability that a novel isolate found in the next collection originated from sexual reproduction. Due to the relatively small number of isolates sampled from each plot at each time point, allele frequencies at each time point were estimated by pooling all isolates from different replications and host treatments. For example, allele frequencies based on all inoculated plots in 2004A were used to calculate the probability that a novel isolate found in the 2004B collection originated from sexual reproduction. A small value (one divided by twice the total number of isolates collected from the entire experiment) $(2 n=2,572)$ was used to calculate the prior probability of a novel isolate being a recombinant if the isolate had an allele that was not present in the earlier collection. The allele frequencies used to calculate the probability of novel isolates originating from immigration were estimated using only the novel isolates sampled from the noninoculated plots.
The relative proportions of novel genotypes originating via sexual reproduction among fungal isolates within the inoculated plots or via immigration from outside the inoculated plots was estimated using a maximum likelihood approach described previously (63) under the assumption that the contribution of mutation to the contemporary populations was negligible (i.e., all of the novel isolates detected in the inoculated plots originated from immigration and recombination events). The unknown parameters in the formula (63) were solved by using the Goal Seek function in Microsoft Excel 2003. Only alleles that could be scored unambiguously and novel isolates that had complete MLHT data across the 11 loci were included in this analysis. Confidence intervals for the estimated immigration and recombination rates were calculated using bootstrapping with 1,000 repetitions.

The posterior probability of a novel genotype resulting from immigration or sexual recombination between the inoculants or between inoculants and immigrants was calculated by using the estimated immigration rate as a prior based on the formula

$$
\frac{m p_{G_{x}^{I}}}{m p_{G_{x}^{I}}+(1-m) p_{G_{x}^{R}}}
$$

where $P_{G_{x}^{R}}$ and $P_{G_{x}^{I}}$ represent the prior probability of a novel isolate being recombinant and immigrant, respectively (62) and $m$ is the maximum likelihood estimate of immigration rate. The formula differs slightly from previous ones $(39,41,62)$ because it reflects the prior informative knowledge of immigration rates. Formulae used previously were derived under the condition of a noninformative prior regarding immigration rates and assumed that immigration rates and recombination rates were equal (i.e., $m=r=0.50$.

Isolates with posterior probabilities $\geq 90 \%$ of being recombinants were assigned to the recombinant category. Isolates with posterior probabilities $\geq 90 \%$ of being immigrants were assigned to the immigrant category. Isolates with posterior probabilities of 10 to $90 \%$ of being immigrants or recombinants were assigned to the uncertain category (62).

\section{RESULTS}

Disease development. In 2004, the weather was drier than normal, presenting suboptimal conditions for infection. There was only $135 \mathrm{~mm}$ of precipitation between 1 March and 30 June. As a consequence, the amount of disease in 2004 was below average for the entire season. In 2005, the total precipitation during the same period of time was $282 \mathrm{~mm}$ and the weather conditions were conducive for epidemic development of Stagonospora nodorum blotch, resulting in a greater number of earlier infections. The majority of infections occurred on leaves. Glume infections were rare in both years. Disease was also detected in the control plots but at a lower incidence compared with the inoculated plots.

Recovery of inoculants and novel genotypes. In total, 1,286 isolates were assayed for the 1 minisatellite marker and 10 microsatellite markers. Among them, 1,187 isolates were recovered from the inoculated plots and the remaining 99 isolates were recovered from the noninoculated control plots. In the samples recovered from the inoculated plots, 637 isolates had MLHTs matching the nine released isolates and were considered to be the asexual progeny of the inoculants (Table 2). The other 550 isolates had MLHTs differing from the nine inoculants and were treated as novel genotypes. In the samples recovered from the noninoculated control plots, only 13 isolates had MLHTs matching four of the nine released isolates while the remaining 86 isolates had MLHTs differing from the nine inoculants. The nine inoculants were not equally established across the experimental plots and only eight of them were recovered.

The majority of the novel genotypes were detected only once. In all, 9 novel genotypes ( 4 in 2004A and 5 in 2004B) were 
detected more than once in the first year of the experiment, whereas 20 novel genotypes (13 in 2005A and 7 in 2005B) were detected more than once in the second year of the experiment. Novel isolates sharing the same genotype were usually recovered from the same plot at the same point in time. In three cases (two in 2005A and one in 2005B), novel isolates with the same genotypes were recovered from adjacent plots at the same point in time. The most frequent novel genotype was detected five times from two adjacent plots in 2005B. The average frequency of novel isolates in the inoculated plots increased steadily from $14 \%$ in the first collection to $75 \%$ in the fourth collection (Fig. 1). This pattern was found across all host treatments (data not shown).

Detection of novel alleles. Novel alleles, defined as alleles not present in the inoculants, appeared in both noninoculated control and inoculated plots. The mean number of novel alleles in the

TABLE 2. Total number of isolates assayed with molecular markers, number of isolates with multilocus haplotypes matching the released genotypes (inoculants), and number of novel isolates and genotypes with multilocus haplotypes differing from the released genotypes

\begin{tabular}{lcccc}
\hline & & & \multicolumn{2}{c}{ No. of novel isolates } \\
\cline { 4 - 5 } Collections & Total isolates & Inoculants & Isolates & Genotypes \\
\hline Inoculated plots & & & & \\
2004A & 223 & 192 & 31 & 20 \\
2004B & 362 & 224 & 138 & 104 \\
2005A & 317 & 150 & 167 & 132 \\
2005B & 285 & 71 & 214 & 186 \\
Control plots & 99 & 13 & 86 & 74 \\
Total & 1,286 & 650 & 636 & 496 \\
\hline
\end{tabular}

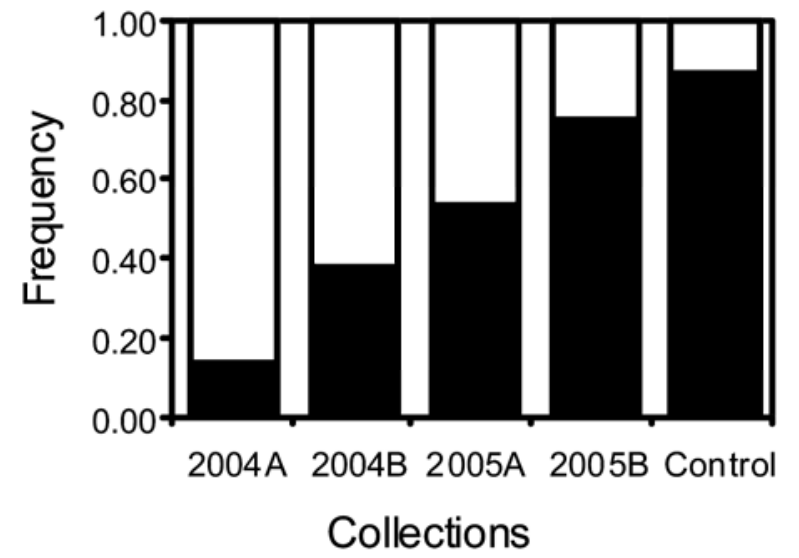

Fig. 1. Changes in frequencies of novel and inoculated isolates over time in the experimental Phaeosphaeria nodorum populations. Frequencies of novel isolates are shown in black. fungal populations recovered from the inoculated plots steadily increased from 2.55 per locus in 2004A to 4.23 per locus in 2005A but decreased slightly to 3.93 per locus in 2005B (Table $3)$. When the isolates from different collections were combined, the mean number of novel alleles detected in the inoculated plots was similar to that detected in the noninoculated control plots (Table 3, columns 6 and 7). The percentage of novel isolates carrying novel alleles steadily increased from $35 \%$ in the first collection to $57 \%$ in the last collection. Among the 86 novel isolates found in the noninoculated plots, 63 (73\%) of them carried novel alleles.

Difference in allele frequency between the novel isolates recovered from the inoculated plots and noninoculated control plots. The number of loci at which allele frequencies differed between the novel isolates from noninoculated control and inoculated plots steadily increased over time (Table 4). Only 1 of 11 loci differed significantly in allele frequency when the sample from the noninoculated control plots was compared with that from the first collection (2004A) in inoculated plots. The number of loci with significant differences increased to six, seven, and eight when the sample from the noninoculated control plots was compared with that from the second (2004B), third (2005A), and fourth (2005B) samples from the inoculated plots, respectively. For three loci (SN8, SN11, and SN23), allele frequencies did not differ in any pairwise comparison.

Genotypic diversity and test for random mating. Genotypic diversity in the novel isolates sampled from the inoculated plots steadily increased during the course of the experiment (Table 5). Brown's analysis of multilocus associations revealed lower than expected variances of heterozygosity in all $P$. nodorum populations composed only of novel isolates (Table 5), suggesting an

TABLE 4. Values for contingency $\chi^{2}$ tests for homogeneity in allele frequencies between the novel isolates recovered from the inoculated and noninoculated control plots ${ }^{\mathrm{a}}$

\begin{tabular}{lcccc}
\hline & \multicolumn{4}{c}{ Control versus } \\
\cline { 2 - 5 } SSR locus & $2004 \mathrm{~A}$ & $2004 \mathrm{~B}$ & $2005 \mathrm{~A}$ & $2005 \mathrm{~B}$ \\
\hline SN15 & $5.32(3)$ & $7.33(3)$ & $5.22(4)$ & $13.22(3)^{*}$ \\
SN3 & $2.45(1)$ & $10.24(2)^{* *}$ & $7.01(2)^{*}$ & $16.60(2)^{* *}$ \\
SN11 & $0.56(1)$ & $2.27(3)$ & $2.70(3)$ & $5.79(2)$ \\
SN5 & $8.19(7)$ & $50.41(9)^{* *}$ & $55.93(9)^{* *}$ & $54.29(9)^{* *}$ \\
SN1 & $0.09(3)$ & $18.67(8)^{*}$ & $34.30(8)^{* *}$ & $29.01(7)^{* *}$ \\
SN17 & $5.33(5)$ & $30.98(9)^{* *}$ & $32.63(8)^{* *}$ & $47.31(8)^{* *}$ \\
SN8 & $3.09(2)$ & $4.88(3)$ & $5.06(4)$ & $5.94(3)$ \\
SN22 & $12.86(7)$ & $55.26(9)^{* *}$ & $53.67(8)^{* *}$ & $51.84(7)^{* *}$ \\
SN21 & $21.10(6)^{* *}$ & $50.28(6)^{* *}$ & $34.75(6)^{* *}$ & $59.43(6)^{* *}$ \\
SN23 & $0.28(1)$ & $1.76(3)$ & $0.842(2)$ & $0.01(1)$ \\
SN16 & $5.52(3)$ & $10.81(5)$ & $13.99(3)^{*}$ & $22.79(3)^{* *}$ \\
\hline
\end{tabular}

a Numbers in parenthesis are the degrees of freedom; * and ** indicate significant at $P=0.05$ and 0.01 levels, respectively.

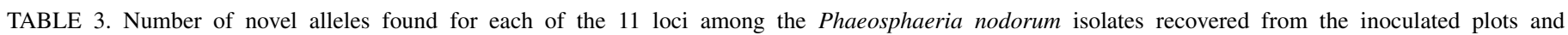
noninoculated control plots over the course of the experiment ${ }^{\mathrm{a}}$

\begin{tabular}{|c|c|c|c|c|c|c|}
\hline \multirow[b]{2}{*}{ SSR locus } & \multicolumn{5}{|c|}{ Inoculated plots } & \multirow[b]{2}{*}{ Control } \\
\hline & $2004 \mathrm{~A}$ & 2004B & $2005 \mathrm{~A}$ & $2005 B$ & Total & \\
\hline SN15 & 3 & 4 & 5 & 4 & 5 & 3 \\
\hline SN3 & 1 & 2 & 3 & 3 & 3 & 3 \\
\hline SN11 & 1 & 2 & 3 & 3 & 3 & 3 \\
\hline SN5 & 3 & 5 & 7 & 6 & 9 & 10 \\
\hline SN1 & 4 & 5 & 5 & 4 & 5 & 7 \\
\hline SN17 & 3 & 4 & 4 & 4 & 4 & 9 \\
\hline SN8 & 2 & 2 & 2 & 3 & 3 & 4 \\
\hline SN22 & 3 & 5 & 7 & 5 & 9 & 8 \\
\hline SN21 & 4 & 5 & 5 & 5 & 5 & 7 \\
\hline SN23 & 2 & 4 & 3 & 2 & 5 & 2 \\
\hline SN16 & 2 & 3 & 3 & 4 & 5 & 4 \\
\hline Average & $2.55( \pm 0.23)$ & $3.73( \pm 0.11)$ & $4.23( \pm 0.31)$ & $3.91( \pm 0.27)$ & $5.09( \pm 0.35)$ & $5.45( \pm 0.27)$ \\
\hline
\end{tabular}

a Confidence intervals for the novel alleles were generated using bootstrapping with 1,000 repetitions; numbers in parentheses indicate $95 \%$ confidence intervals. 
absence of significant associations among loci. The parsimony tree length permutation test also did not reject the hypothesis of random mating among the loci in the novel isolates (Table 5).

Estimating immigration or recombination rates and determining the origin of each novel genotype. The proportion of $P$. nodorum isolates derived from recombination in the inoculated plots steadily increased over time (Table 6). In the second collection $(2004 \mathrm{~B}), \approx 30 \%$ of isolates in the $P$. nodorum population recovered from the inoculated plots were most likely to be recombinants. By the end of the experiment (2005B), recombinants made up nearly three-quarters of the population recovered from the inoculated plots. The estimated proportion of immigrants in the inoculated plots increased from $9 \%$ in $2004 \mathrm{~B}$ to $16 \%$ in $2005 \mathrm{~A}$ but dropped to $7 \%$ in 2005B. The effect of host genotype on the balance among sexual reproduction, asexual reproduction,

TABLE 5. Genotypic diversity (GD) and tests for random mating among the novel isolates of Phaeosphaeria nodorum recovered from the control and inoculated plots over time ${ }^{\mathrm{a}}$

\begin{tabular}{lllllll}
\hline & & \multicolumn{2}{c}{ Brown's test } & & \multicolumn{2}{c}{ PTLPT $^{\mathrm{e}}$} \\
\cline { 3 - 4 } \cline { 6 - 7 } Collection & $\mathrm{GD}^{\mathrm{b}}$ & $S_{k}{ }^{2 \mathrm{c}}$ & $\mathrm{L}_{2}{ }^{\mathrm{d}}$ & & $\mathrm{L}$ & \\
\hline 2004A & 0.33 & $\ldots$ & $\ldots$ & & $\ldots$ & $\ldots$ \\
2004B & 0.40 & 0.5 & 1.4 & & 186 & 0.52 \\
$2005 \mathrm{~A}$ & 0.52 & 0.4 & 1.2 & & 175 & 0.90 \\
2005B & 0.74 & 0.3 & 1.0 & & 282 & 0.58 \\
Control & 0.58 & 0.8 & 1.5 & & 181 & 0.10 \\
\hline
\end{tabular}

${ }^{a}$ Measures of gametic equilibrium were not estimated for the 2004A collections because of the small sample sizes.

b Standardized Stoddart and Taylor index.

c Observed variance of the number of heterozygous comparisons calculated using Brown's multilocus association (8).

d Upper $95 \%$ confidence limit of $S_{k}^{2}$.

e PTLPT = parsimony tree length permutation test (28). Null hypothesis of random mating was tested by comparing the length of the observed parsimony tree $(\mathrm{L})$ to the lengths of 1,000 randomized trees generated from artificially recombined data sets.

TABLE 6. Proportions of Phaeosphaeria nodorum isolates in the inoculated plots originating from asexual reproduction of released isolates (inoculants), immigration from outside experimental plots (immigrants), and sexual recombination among existing isolates within the experimental plots (recombinants) ${ }^{\mathrm{a}}$

\begin{tabular}{lccc}
\hline Collection & Inoculants & Immigrants & Recombinants \\
\hline $2004 \mathrm{~A}^{\mathrm{b}}$ & 0.86 & $\ldots$ & $\ldots$ \\
$2004 \mathrm{~B}$ & 0.62 & $0.09( \pm 0.03)$ & $0.29( \pm 0.03)$ \\
$2005 \mathrm{~A}$ & 0.47 & $0.16( \pm 0.04)$ & $0.37( \pm 0.04)$ \\
$2005 \mathrm{~B}$ & 0.25 & $0.07( \pm 0.02)$ & $0.68( \pm 0.02)$ \\
\hline
\end{tabular}

${ }^{a}$ Results were derived using a combination of microsatellite assays, a maximum likelihood approach (63), and bootstrapping. Bootstrap means for immigrants in $2004 \mathrm{~B}, 2005 \mathrm{~A}$, and $2005 \mathrm{~B}$ were $0.08,0.18$ and 0.9 , respectively, and the bootstrap means for recombinants in $2004 \mathrm{~B}, 2005 \mathrm{~A}$, and $2005 \mathrm{~B}$ were $0.30,0.35$, and 0.66 , respectively. Data in parenthesis are $95 \%$ confidence intervals.

b Proportions of immigrants and recombinants could not be calculated for the first collection because the MLE approach requires frequency data from an earlier point in time. and immigration could not be determined due to the small sample sizes from each plot.

Using the estimated immigration and recombination rates shown in Table 6 as priors, 316 novel isolates recovered from the inoculated plots across the last three collections were assigned to the recombinant category while only 52 novel isolates were assigned to the immigrant category (Table 7). More than a quarter $(n=151)$ of the novel isolates could not be assigned either to the immigrant category or to the recombinant category with $\geq 90 \%$ confidence and, thus, were placed into the uncertain category.

\section{DISCUSSION}

We conducted a 2-year mark-release-recapture experiment to investigate the relative contributions of immigrants, sexual recombinants, and inoculants to epidemics of Stagonospora nodorum blotch caused by $P$. nodorum. Ascocarps of the pathogen have been observed in many parts of the world $(3,4,13,14,19$, $23,32,38,43)$. In Switzerland, we also found ascocarps in several wheat fields (B. A. McDonald, unpublished data). The evolutionary and epidemiological importance of sexual reproduction relies on both the quantity of sexual offspring produced and the fitness of recombinants relative to immigrants and inoculants. In this study, instead of counting the number of ascocarps formed on various hosts, we used a more efficient population genetic approach to quantify the contribution of sexual reproduction to the population genetic structure and epidemiology of P. nodorum. This approach took into account both the amount of sexual reproduction occurring in $P$. nodorum populations and the fitness of recombinants relative to the immigrants and inoculants that also contributed to the epidemic. Our results revealed that all three sources of inoculum made significant contributions to the epidemic of $P$. nodorum. In all, $51 \%$ of isolates recovered from the inoculated plots had MLHTs matching the nine marked inoculants and could be classified as asexual progeny of the inoculants. The remaining $49 \%$ of recovered isolates had MLHTs differing from the released isolates. A significant number of noninoculated genotypes (43\%) were also recovered in a markrelease-recapture experiment conducted in North America (5).

In addition to wheat, $P$. nodorum may infect a wide range of other gramineous species (49) and could be transmitted through infected wheat seed $(5,44)$. However, fungal isolates recovered from these perennial grasses usually displayed a strong host specialization (45) and did not adapt well to cultivated wheat (26). Because we initiated our field experiment using fungicidetreated seed and the plots were planted into fields that had not been planted with wheat or other hosts of $P$. nodorum for several years, we believe that the possibility that infected seed or stubble were sources of novel isolates was negligible. The novel isolates found in our experiment most likely originated through recombination or immigration. We have several lines of evidence supporting this assumption: (i) there were significant differences in allele frequencies among the populations sampled from the noninoculated control plots and the inoculated plots, (ii) high levels of genotype diversity (little clonality) existed in the populations of

TABLE 7. Contribution of different source categories to the population composition of Phaeosphaeria nodorum sampled from the inoculated plots ${ }^{\mathrm{a}}$

\begin{tabular}{|c|c|c|c|c|c|}
\hline \multirow[b]{2}{*}{ Collection } & \multirow[b]{2}{*}{ Inoculants } & \multicolumn{4}{|c|}{ No. of novel isolates (\%) } \\
\hline & & Recombinants & Uncertain & Immigrants & Sum \\
\hline 2004A & $192(86)$ & $\ldots$ & $\ldots$ & $\ldots$ & $31(14)$ \\
\hline 2004B & $224(72)$ & $82(23)$ & 45 (12) & $11(3)$ & $138(38)$ \\
\hline $2005 \mathrm{~A}$ & $150(47)$ & $84(27)$ & $53(17)$ & $30(9)$ & $167(53)$ \\
\hline 2005B & $71(25)$ & $150(52)$ & 53 (19) & $11(4)$ & $214(75)$ \\
\hline Total & 637 & 316 & 151 & 52 & 550 \\
\hline
\end{tabular}

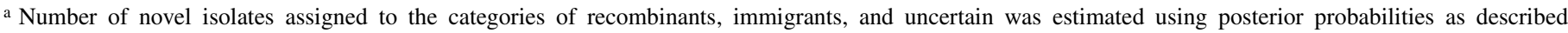
previously (62). Data in parenthesis are the overall proportions of inoculants and novel isolates (recombinants, uncertain, and immigrants) across all isolates (novel + inoculated). 
novel isolates from both inoculated and control plots, (iii) the populations of novel isolates in both inoculated and control plots were at gametic equilibrium, (iv) there was a steady increase in the frequency of novel genotypes over the course of the experiment, and (v) there was a steady increase in the number of novel alleles over the course of the experiment.

The noninoculated control plots allowed us to detect possible seedborne sources of inoculum. If the main source of novel primary inoculum had been infected seed, we would expect to find no differences in allele frequencies among the novel isolates recovered from the inoculated and noninoculated control plots throughout the course of the experiment. Instead, we found significant differences in allele frequencies that increased over the course of the experiment (Table 4). We also found much less disease in the noninoculated plots compared with inoculated plots in both years. Disease was either absent or at a very low level in the noninoculated plots in the 2003-04 season, with the result that only 0-4 isolates could be obtained from the control plots in the 2004A and 2004B collections. In contrast, a significant number of infected leaves were found throughout the inoculated plots during the same season. Because the artificially infected plots were separated by the nonhost triticale, the splash-dispersed pycnidiospores would need to travel across a distance of at least $1.5 \mathrm{~m}$ to infect the control plots. The failure to find any released genotypes in the control plots in the first year indicates that few or no pycnidiospores traveled further than $1.5 \mathrm{~m}$ during the growing season. We hypothesize that inoculants found in the control plots in the second year of the experiment were introduced via infected straw that blew into the control plots.

Analyses of allelic associations and genotype diversity are routinely used to infer the reproductive strategies of plant pathogens $(17,21,40,52,65)$. The lack of allelic associations coupled with high levels of genotype diversity is generally considered a hallmark of sexual recombination. Two independent methods failed to detect disequilibrium in the novel isolate populations, consistent with our hypothesis that the novel isolates originated from a random mating population and signifying an important role for ascospores in the epidemic of Stagonospora nodorum blotch. The high levels of genotype diversity and the gradual increase in the proportion of novel isolates found in inoculated plots also support the idea that immigrants and recombinants play an important role in the epidemiology of this pathogen. The average frequency of novel isolates increased from $<20 \%$ in the 2004 A sample to $>75 \%$ in the $2005 B$ sample.

The finding of an increase in the number of novel alleles over time in inoculated plots further supports the hypothesis that an influx of immigrants contributed to the epidemic of Stagonospora nodorum blotch. These novel alleles could have originated through mutation but we consider it unlikely that mutation alone could have generated novel alleles in such a large fraction of the pathogen population within the time frame of this experiment. It is much more likely that isolates carrying novel alleles were either immigrants deposited by ascospore showers that originated from outside the experimental plots or recombinants derived from crosses between immigrants and the released inoculants.

In this experiment, novel isolates could have originated either via airborne ascospores blown into the experimental plots from outside (immigrants) or via recombination among inoculants or immigrants within the experimental plots (recombinants). We believe that novel isolates in the noninoculated control plots and the first collection of the inoculated plots originated from immigration whereas the novel isolates in the late collections of the inoculated plots originated from a combination of immigration and recombination. Statistical tests for differences in allele frequency support our hypothesis. No differences in allele frequency were found between the novel isolates sampled from the noninoculated control plots and inoculated plots at 2004A for 10 of the 11 loci, indicating that the two groups of novel isolates originated from the same source population (i.e., airborne ascospores) at the beginning of epidemic. However, allele frequencies between the two groups diverged significantly over time. Additional analysis with a maximum likelihood approach confirmed that a large proportion of the novel isolates in the inoculated plots at 2004B, 2005A, and 2005B originated from sexual recombination among the isolates existing within the experimental plots. The proportion of recombinants in the inoculated plots increased from $29 \%$ in the second collection (2004B) to $65 \%$ in the final collection (2005B) (Table 6). Further analyses using Bayesian theory indicated that 82,80 , and 154 isolates in the 2004B, $2005 \mathrm{~A}$, and 2005B samples, respectively, could be assigned to the recombinant category with $\geq 90 \%$ probability. These results provide strong support for the hypothesis that sexual recombinants originating from within experimental plots during the growing season made a significant contribution to the development of these leaf blotch epidemics.

In all, $<20 \%$ of the novel $P$. nodorum isolates originated from immigration and there was a decrease in the contribution of immigrants to the epidemic from 2005A to 2005B. In the maximum likelihood approach we developed (63), all isolates, including immigrants, are used to estimate allele frequencies in sexual populations. Thus, our estimates of recombination rates include mating among inoculants as well as between immigrants and inoculants. This procedure could lead to underestimation of actual immigration rates. The ability to differentiate between immigrants and recombinants diminishes over time because the allele frequencies in the inoculated plots steadily approach the allele frequencies in the immigrant population as a result of constant immigration over time. This could explain why the proportion of isolates that could be assigned as immigrants with $\geq 90 \%$ confidence declined substantially in the 2005B sample while the proportion of isolates that could not be assigned increased over time (Table 7).

The finding of 650 isolates with MLHTs matching the 9 marked isolates confirms that asexual reproduction also contributed significantly to the epidemic. By applying the crop residue collected at the end of the 2004 season to initiate the 2005 epidemic, we were able to corroborate in a quantitative way the earlier findings (20) that crop residue can play an important role in initiating new epidemics. The recovery of a high proportion of isolates (47\%) with MLHT matching the inoculants in the inoculated plots in $2005 \mathrm{~A}$ illustrates that $P$. nodorum isolates have the potential to persist on crop residue between growing seasons. However, despite providing optimal conditions for asexual carryover by storing the bags containing the infected straw in a protected place, the frequencies of the inoculants decreased sharply over time and they were rapidly replaced between spring and summer by novel genotypes that originated through recombination or immigration. Thus, we conclude that asexual lineages of $P$. nodorum are unlikely to persist across many seasons in farmer's fields.

The statistical power associated with the maximum likelihood estimates of recombination or immigration rates can be affected by sample size $(9,63)$. In our experiment, only 86 individuals were used to calculate allele frequencies in the immigrant population. Although this sample size may have been suboptimal for this type of analysis, we do not believe that it affected our conclusions. When we used a larger Swiss population (191 isolates) collected earlier (53) to estimate the allele frequencies in the immigrant population, we came to a result that differed by only $2 \%$ regarding the relative contributions of recombinants and immigrants to the population genetic structure of the pathogen (data not shown).

This mark-release-recapture study provided novel and valuable insight into the relative contributions of immigrants, sexual recombinants, and inoculants to epidemics of $P$. nodorum. Infected seed have been proposed to be one of the main sources of 
inoculum leading to the initiation of epidemics of Stagonospora nodorum blotch in wheat $(5,44)$. To increase the statistical power to detect novel genotypes, we used fungicide-treated seed to minimize the effect of seedborne infection in the current study. As a consequence, we were unable to directly quantify the contribution of seedborne infection to the epidemiology and population genetic structure of the pathogen. Our findings indicate that windblown ascospores can provide important sources of both primary and secondary inoculum. Although this experiment was not designed to measure long distance movement of ascospores, we consider it likely that ascospores can move over distances of at least a few kilometers and perhaps much further. Ascospores provide windborne inoculum that can be transmitted to neighboring fields, both during and between growing seasons. Thus, even wheat fields that have experienced the best sanitation practices, including multi-year crop rotation (to eliminate infected straw from previous crops) and planted with certified disease-free seed or seed treated with fungicides, can be infected by ascospore showers that originate from neighboring fields. The potential for long-distance dissemination of airborne ascospores also increases the risk for rapid dissemination of genotypes carrying novel virulence or fungicide resistance alleles. Although we showed that the inoculants carried on infected tissues can persist between generations and even seasons, there was a rapid decline in the frequency of the inoculants over the course of the experiment. This suggests that clones are unlikely to persist over many years. On the contrary, we predict that populations will continuously change as new combinations of alleles come together through recombination events. Because of the important contribution made by airborne propagules to the epidemiology of this pathogen, control strategies should be considered on a more regional basis (36), as is the case with rust and powdery mildew diseases (58).

\section{ACKNOWLEDGMENTS}

This research was supported by the Swiss Federal Institute of Technology Grant TH-49a/02-1. Microsatellite and minisatellite data were collected using facilities of the Genetic Diversity Center at ETH Zurich. We thank S. Kellenberger for field work; P. Brunner and P. L. Zaffarano for help with the data analysis; and V. Martinez, C. Phan, S. Seeholzer, and M. Zala for technical assistance.

\section{LITERATURE CITED}

1. Abang, M. M., Baum, M., Ceccarelli, S., Grando, S., Linde, S., Yahyaoui, A., Zhan, J., and McDonald, B. A. 2006. Differential selection on Rhynchosporium secalis during the parasitic and saprophytic phases in the barley scald disease cycle. Phytopathology 96:1214-1222.

2. Adhikari, T. B., Ali, S., Burlakoti, R. R., Singh, P. K., Mergoum, M., and Goodwin, S. B. 2008. Genetic structure of Phaeosphaeria nodorum populations in the North-Central and Midwestern United States. Phytopathology 98:101-107.

3. Arseniuk, E., Góral, T., and Scharen, A. L. 1998. Seasonal patterns of spore dispersal of Phaeosphaeria nodorum spp. and Stagonospora spp. Plant Dis. 82:187-194.

4. Bathgate J. A., and Loughman, R. 2001. Ascospores are a source of inoculum of Phaeosphaeria nodorum, $P$. avenaria $\mathrm{f}$. sp. avenaria and Mycosphaerella graminicola in Western Australia. Australas. Plant Pathol. 30:317-322

5. Bennett, R. S., Milgroom, M. G., Sainudiin, R., Cunfer, B. M., and Bergstrom, G. C. 2007. Relative contribution of seed-transmitted inoculum to foliar populations of Phaeosphaeria nodorum. Phytopathology 97:584-591.

6. Bennett, R. S., Yun, S. H., Lee, T. Y., Turgeon, B. G., Arseniuk, E., Cunfer, B. M., and Bergstrom, G. C. 2003. Identity and conservation of mating type genes in geographically diverse isolates of Phaeosphaeria nodorum. Fungal Genet. Biol. 40:25-37.

7. Blixt, E., Olson, Å., Högberg, N., Djurle, A., and Yuen J. 2008. Mating type distribution and genetic structure are consistent with sexual recombination in the Swedish population of Phaeosphaeria nodorum. Plant Pathol. 57:634-641.
8. Brown, A. H. D., Feldman, M. W., and Nevo, E. 1980. Multilocus structure of natural populations of Hordeum spontaneum. Genetics 96:523-526.

9. Brown, J. K. M. 2000. Estimation of rates of recombination and migration in populations of plant pathogens. Phytopathology 90:320-323.

10. Brunner, P. C., Stefanato, F. L., and McDonald, B. A. 2008. Evolution of the CYP51 gene in Mycosphaerella graminicola: evidence for intragenic recombination and selective replacement. Mol. Plant Pathol. 305-316.

11. Burt, A., Carter, D. A., Koenig, G. L., White, T. J., and Taylor, J. W. 1996. Molecular markers reveal cryptic sex in the human pathogen Coccidioides immitis. Proc. Natl. Acad. Sci. USA 93:770-773.

12. Carter, M. V., and Moller, W. J. 1961. Factors affecting the survival and dissemination of Mycosphaerella pinodes (Berk. and Blox.) vestergr. in South Australian irrigated pea fields. Aust. J. Agric. Res. 12:878-888.

13. Cowger, C., and Silva-Rojas, H. V. 2006. Frequency of Phaeosphaeria nodorum, the sexual stage of Stagonospora nodorum, on winter wheat in North Carolina. Phytopathology 96:860-866.

14. Duczek, L. J., Sutherland, K. A., Reed, S. L., Bailey, K. L., and Lafond, G. P. 1999. Survival of leaf spot pathogens on crop residues of wheat and barley in Saskatchewan. Can. J. Plant Pathol. 21:165-173.

15. Everitt, B. S. 1977. Pages 1-37 in: The Analysis of Contingency Tables. Wiley, New York.

16. Grunwald, N. J., Goodwin, S. B., Milgroom, M. G., and Fry, W. E. 2003. Analysis of genotypic diversity data for populations of microorganisms. Phytopathology 93:738-746.

17. Grunwald, N. J., and Hoheisel, G. A. 2006. Hierarchical analysis of diversity, selfing, and genetic differentiation in populations of the oomycete Aphanomyces euteiches. Phytopathology 96:1134-1141.

18. Heald F. D., Gardner M. W., and Studhalter R. A. 1915. Air and wind dissemination of ascospores of chestnut-blight fungus. J. Agric. Res. 3:493-526.

19. Hewett, P. D. 1975. Septoria nodorum on seedlings and stubble of winterwheat Trans. Br. Mycol. Soc. 65:7-18.

20. Holmes, S. J. I., and Colhoun, J. 1975. Straw-borne inoculum of Septoria nodorum and S. tritici in relation to incidence of disease on wheat plants. Plant Pathol. 24:63-66.

21. Keller, S. M., McDermott, J. M., Pettway, R. E., Wolfe, M. S., and McDonald B. A. 1997. Gene flow and sexual reproduction in the wheat glume blotch pathogen Phaeosphaeria nodorum, (anamorph Stagonospora nodorum). Phytopathology 87:353-358.

22. Keller, S. M., Wolfe, M. S., McDermott, J. M., and McDonald B. A. 1997. High genetic similarity among populations of Phaeosphaeria nodorum across wheat cultivars and regions in Switzerland. Phytopathology 87:1134-1139.

23. Kemp, G. H. J., Pretorius, Z. A., and LeRoux, J. 1989. First report of the teleomorph of Leptosphaeria nodorum on wheat in South Africa. Mycol. Res. 98:114-115.

24. King, J. E., Cook, R. J., and Melville, S. C. 1983. A review of Septoria diseases of wheat and barley. Ann. Appl. Biol. 103:3b-373.

25. Koufopanou, V., Burt, A., and Taylor, J. W. 1997. Concordance of gene genealogies reveals reproductive isolation in the pathogenic fungus Coccidioides immitis. Proc. Natl. Acad. Sci. USA 94:5478-5482.

26. Krupinsky, J. M. 1997. Stability of Stagonospora nodorum isolates from perennial grass hosts after passage through wheat. Plant Dis. 81:10371041.

27. LoBuglio, K. F., and Taylor, J. W. 2002. Recombination and genetic differentiation in mycorrhizal fungus Cenoccocum geophilum Fr. Mycologia 94:772-780.

28. Maynard Smith, J., Smith, N. H., O'Rourke, M., and Spratt, B. G. 1993. How clonal are bacteria? Proc. Natl. Acad. Sci. USA 90:4384-4388.

29. McDonald, B. A., and Linde, C. 2002. Pathogen population genetics, evolutionary potential and durable resistance. Annu. Rev. Phytopathol. 40:349-379.

30. McDonald, B. A., and Martinez, J. P. 1991. DNA fingerprinting of the plant pathogenic fungus Mycosphaerella graminicola (anamorph Septoria tritici). Exp. Mycol. 15:146-158.

31. McDonald, B. A., Miles, J., Nelson, L. R., and Pettway, R. E. 1994. Genetic variability in nuclear DNA in field populations of Stagonospora nodorum. Phytopathology 84:250-255.

32. Mehta, Y. R. 1975. Leptosphaeria nodorum on wheat in Brazil and its importance. Plant Dis. Rep. 59:404-406.

33. Milgroom, M. G. 1996. Recombination and the multilocus structure of fungal populations. Annu. Rev. Phytopathol. 34:457-477.

34. Milgroom, M. G., and Peever, T. L. 2003. Population biology of plant pathogens - the synthesis of plant disease epidemiology and population genetics. Plant Dis. 87:608-617.

35. Mittelstädt, A., and Fehrmann, H. 1987. The occurrence of the perfect stage of Septoria nodorum in the Federal Republic of Germany. Z. Pflanzenkrankh. Pflanzenschutz 94:380-385.

36. Mundt, C. C., Cowger, C., and Garret, K. A. 2002. Relevance of inte- 
grated disease management to resistance durability. Euphytica 124:245252 .

37. O'Donnell, K., Kistler, H. C., Tacke, B. K., and Casper, H. H. 2000. Gene genealogies reveal global phylogeographoic structure and reproductive isolation among lineages of Fusarium graminearum, the fungus causing wheat scab. Proc. Natl. Acad. Sci. USA 97:7905-7910.

38. O'Reilly, P., Bannon, E., and Downes, M. J. 1988. Leptosphaeria nodorum on wheat in Ireland. Plant Pathol. 37:153-154.

39. Paetkau, D., Calvert, W., Stirling, I., and Strobeck, C. 1995. Microsatellite analysis of population-structure in Canadian polar bears. Mol. Ecol. 4:347-364.

40. Prospero, S., Grunwald, N. J., Winton, L. M., and Hansen, E. M., 2009. Migration patterns of the emerging plant pathogen Phytophthora ramorum on the west coast of the United States of America. Phytopathology 99:739-749.

41. Rannala, B., and Mountain, J. L. 1997. Detecting immigration by using multilocus genotypes. Proc. Natl. Acad. Sci. USA 94:9197-922.

42. Sabat, A. J., Wladyka, B., Kosowska-Shick, K., Grundmann, H., van Dijl, J. M., Kowal, J., Appelbaum, P. C., Dubin, A., and Hryniewicz, W., 2008. Polymorphism, genetic exchange and intragenic recombination of the aureolysin gene among Staphylococcus aureus strains. BMC Microbiol. 129. DOI: $10.1186 / 1471-2180-8-129$

43. Sanderson, F. R., and Hampton, J. G. 1978. Role of perfect states in the epidemiology of the common Septoria diseases of wheat. N. Z. J. Agric. Res. 21:277-281.

44. Shah, D. A, Bergstrom, G. C., and Ueng, P. P. 1995. Initiation of Septoria nodorum blotch epidemics in winter wheat by seed borne Stagonospora nodorum. Phytopathology 85:1463-1468.

45. Shearer, B. L., and Zadoks, J. C. 1971. Observations on the host range of an isolate of Septoria nodorum from wheat. Eur. J. Plant Pathol. 78:153159.

46. Solomon, P. S., Parker, K., Loughman, R., and Oliver, R. P. 2004. Both mating types of Phaeosphaeria (anamorph Stagonospora) nodorum are present in Western Australia. Eur. J. Plant Pathol. 110:763-766.

47. Sommerhalder, R. J., McDonald, B. A., and Zhan, J. 2006. The frequencies and spatial distribution of mating types in Stagonospora nodorum are consistent with recurring sexual reproduction. Phytopathology 96:234-239.

48. Sommerhalder, R. J., McDonald, B. A., and Zhan, J. 2007. Concordant evolution of mitochondrial and nuclear genomes in the wheat pathogen Phaeosphaeria nodorum. Fungal Genet. Biol. 44:764-772.

49. Sprague, R. 1950. Diseases of Cereals and Grasses in North America. Ronald Press Co., New York.

50. Stensvand, A., Amundsen, T., Semb, L., Gadoury, D. M., and Seem, R. C. 1998. Discharge and dissemination of ascospores by Venturia inaequalis during dew. Plant Dis. 82:761-764.

51. Stoddart, J. A., and Taylor, J. F. 1988. Genotypic diversity: estimation and prediction in samples. Genetics 118:705-711.

52. Stukenbrock, E. H., Banke, S., and McDonald, B. A. 2006. Global migration patterns in the fungal wheat pathogen Phaeosphaeria nodorum. Mol. Ecol. 15:2895-2904.

53. Stukenbrock, E., H., Banke, S., Zala, M., McDonald, B. A., and Oliver, R. P. 2005. Isolation and characterization of EST-derived microsatellite loci from the fungal wheat pathogen Stagonospora nodorum. Mol. Ecol. Notes 5:931-933.

54. Swofford, D. L., 1998. PAUP*: Phylogenetic Analysis Using Parsimony* and Other Methods. Sinauer Associates, Sunderland, MA

55. Torriani, S. F. F., Brunner, P. C., McDonald, B. A., and Sierotzki, H. 2008. QoI resistance emerged independently at least four times in European populations of Mycosphaerella graminicola. Pest Manage. Sci. 65:155162.

56. Van der Hoorn, R. A. L., Kruijt, M., Roth, R., Brandwagt, B. F., Joosten, M. H. A. J., and De Wit, P, J, G, M, 2001. Intragenic recombination generated two distinct $\mathrm{Cf}$ genes that mediate AVR9 recognition in the natural population of Lycopersicon pimpinellifolium. Proc. Natl. Acad. Sci. USA 98:10493-10498.

57. Wang, X., Hughes, A. L., Tsukamoto, T., Ando, T., and Kao, T. H. 2001. Evidence that intragenic recombination contributes to allelic diversity of the S-RNase gene at the self-incompatibility (S) locus in Petunia inflate. Plant Physiol. 125:1012-1022.

58. Wolfe, M. S., Brändle, U., Koller, B., Limpert, J. M., McDermott, J. M., Müller, K., and Schaffner, D. 1992. Barley mildew in Europe: population biology and host resistance. Euphytica 63:125-139.

59. Yeh, F. C., Yang, R. C., Boyle, T. B. J., Ye, Z. H., and Mao, J. X. 1999. POPGENE, the user friendly shareware for population genetic analysis. Molecular Biology and Biotechnology Centre, University of Alberta, Canada.

60. Zaffarano, P. L., McDonald, B. A., Zala, M., and Linde, C. C. 2006 Global hierarchical gene diversity analysis suggests the fertile crescent is not the center of origin of the barley scald pathogen Rhynchosporium secalis. Phytopathology 96:941-950.

61. Zhan, J., Mundt, C. C., Hoffer, M. H., and McDonald, B. A. 2002. Local adaptation and effect of host genotype on the evolution of pathogen: an experimental test in a plant pathosystem. J. Evol. Biol. 15:634-647.

62. Zhan, J., Mundt, C. C., and McDonald, B. A. 1998. Measuring immigration and sexual reproduction in field populations of Mycosphaerella graminicola. Phytopathology 88:1330-1337.

63. Zhan, J., Mundt, C. C., and McDonald, B. A. 2000. Estimation of rates of recombination and migration in populations of plant pathogen-a reply. Phytopathology 90:324-326.

64. Zhan, J., Mundt, C. C., and McDonald, B. A. 2007. Sexual reproduction facilitates the adaptation of parasites to antagonistic host environment: evidence from field experiment with wheat-Mycosphaerella graminicola system. Int. J. Parasitol. 37:861-870.

65. Zhan, J., Pettway, R. E., and McDonald, B. A. 2003. The global genetic structure of the wheat pathogen Mycosphaerella graminicola is characterized by high nuclear diversity, low mitochondrial diversity, regular recombination, and gene flow. Fungal Genet. Biol. 38:286-297. 